cinomas, as expected. A risk index - based on the expression of the top 50 genes that are associated with patient survival, determined from a training set of lung adenocarcinomas - predicted survival and identified a group of patients with high-risk stage I disease in an independent testing set of patients. Importantly, this risk index was then shown to predict survival in a completely independent cohort of 62 stage I lung adenocarcinomas.

Beer and co-workers have shown that gene-expression risk profiles, based on gene association with patient survival, can identify stage I lung adenocarcinoma and distinguish between patients at high risk and low risk of recurrent or metastatic disease within this subgroup. Identification of patients with stage I lung adenocarcinoma at high risk of relapse could allow consideration of adjuvant therapy that might help to improve survival.

Ezzie Hutchinson

(2) References and links ORIGINAL RESEARCH PAPER Beer, D. G. et al. Gene-expression profiles predict survival of patients with lung adenocarcinoma. Nature Med. 8, 816-824 (2002)

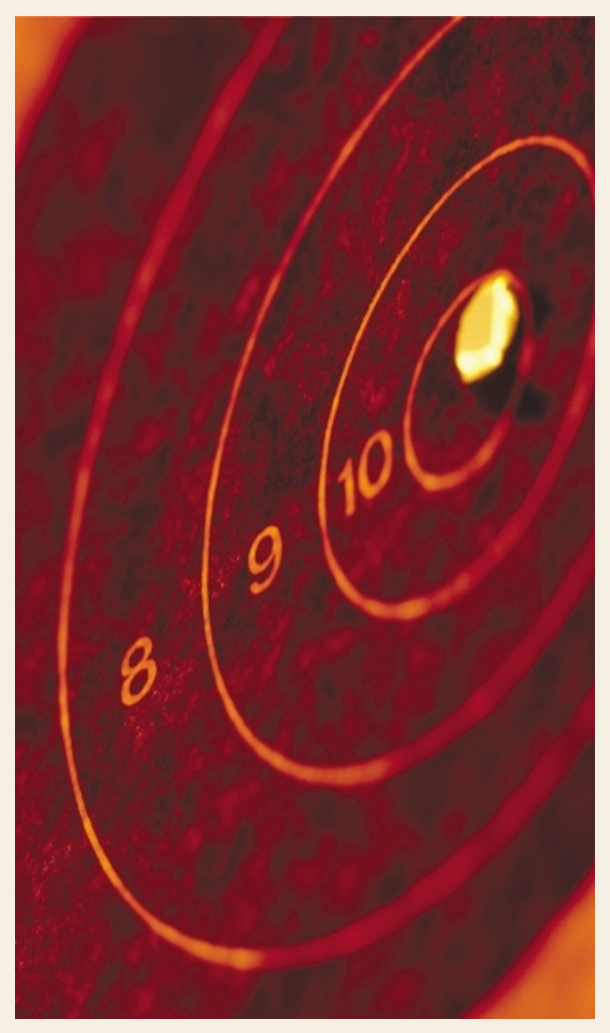

THERAPEUTICS

\section{Will I survive?}

Gloria Gaynor sang "I will survive", but cancer cells might not be quite so lucky, as Dario Altieri and colleagues report that a new approach to cancer treatment - which involves the sequential treatment of two drugs - can induce massive cell death and suppress tumour growth.

Survivin is an inhibitor of apoptosis that is frequently upregulated in cancer. However, it has now been shown to be further upregulated in HeLa cells, following treatment with the chemotherapeutic drug paclitaxel — a microtubule poison that arrests cells at the G2-M transition. Therefore, giving low-dose paclitaxel, combined with a drug that inhibits survivin, might prove more effective and less toxic than the standard single-drug regimen that is used, at present, for several solid tumours.

But how does paclitaxel affect survivin levels? Northern blots confirmed that the mRNA was not increased, so paclitaxel must regulate survivin at the protein level. Survivin is known to be phosphorylated on threonine (Thr) 34 by CDC2 - a cyclin-dependent kinase that is activated in the paclitaxel-induced mitotic block. Could this be involved in regulating survivin? Treating cells with paclitaxel, followed by the CDC2 inhibitor purvalanol A, 16 hours later, suppressed the phosphorylation of survivin. The HeLa cells were also able to escape the mitotic block and proceed into apoptosis, indicating that inhibiting CDC2 leads to the inhibition of survivin.

As might be expected, CDC2-mediated phosphorylation of survivin results in its stabilization. The half-lives of wild-type survivin and a nonphosphorylatable survivin mutant - in which Thr34 is mutated to alanine - were determined by labelling with ${ }^{35} \mathrm{~S}$-methionine and ${ }^{35} \mathrm{~S}$-cysteine, before chasing with normal media and analysing the level of radioactive decay over time. From this, it was shown that the half-life of survivin decreased from 240 minutes to 90 minutes after the phosphorylation site was mutated.

So, sequentially treating cancer cells with paclitaxel, followed by purvalanol $\mathrm{A}$, results in massive apoptosis - $80 \%$ of cells die - as CDC2 is no longer able to stabilize survivin. Importantly, this seems to be independent of cell type and p53 status. In addition to the HeLa cervical carcinoma cells, both MCF-7 breast carcinoma cells and PC3 prostate carcinoma cells undergo apoptosis with this treatment regimen; 553 is wild type in MCF-7 cells, mutated in PC3 cells and functionally inactivated in HeLa cells. Molecular and genetic approaches to inhibiting $\mathrm{CDC} 2$ - using a kinasedead mutant and a conditional $C D C 2$ knockout, respectively - confirmed the requirement for CDC2 in inducing apoptosis.

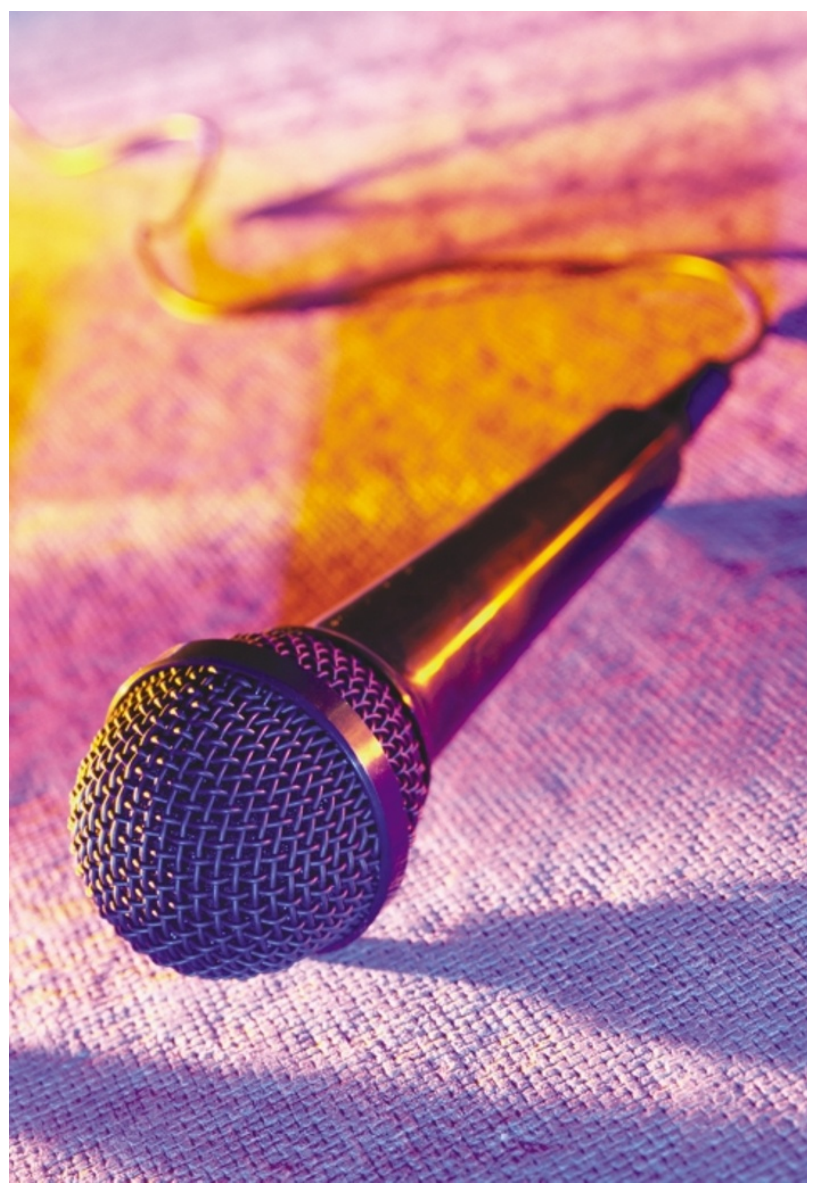

A regimen of paclitaxel, followed by purvalanol A, therefore seems to be a promising therapeutic strategy, but will it prove effective in vivo? A breast cancer xenograft model - in which MCF-7 cells are grown beneath the skin in immunodeficient mice - was used to investigate this. After tumours were established, paclitaxel, purvalanol A, or the sequential treatment of paclitaxel and purvalanol A were administered to the mice. Only the sequential treatment resulted in inhibition of tumour growth and extended the survival of the mice the paclitaxel was given at too low a dose to be effective alone. There was no apparent toxicity.

A similar regimen with paclitaxel and the generic cyclin-dependent kinase inhibitor flavopiridol is more effective than any other combination, and has just completed Phase I trials, but the toxicity of flavopiridol might prove limiting. Altieri and colleagues provide a molecular rationale for the clinical observations with flavopiridol, and suggest that CDC2 might be a more specific and less toxic target.

Emma Greenwood

\section{(D) References and links}

ORIGINAL RESEARCH PAPER O'Connor, D. S. et al. A p34 odc2

survival checkpoint in cancer. Cancer Cell 2, 43-54 (2002)

FURTHER READING Altieri, D. C. The molecular basis and potential

role of survivin in cancer diagnosis and therapy. Trends Mol. Med. $\mathbf{7}$, 542-547 (2001) 